\title{
Día de menarquia, expectativa de fiesta y sesgo de memoria
}

\author{
Carlos Y Valenzuela
}

\author{
Day at menarche, «fiesta» \\ expectancy and «memory bias»
}

Background: The photoperiod and temperature were ruled out as main factors of the annual menarche rhythm. Vacations associate to peaks and study periods to troughs of menarche. Some months show contradictory behavior according to their vacationstudy condition. Aim: To test the hypothesis that menarche occurs mainly in relation to fiesta or celebration (birthdays, holidays, and vacations) expectancies. To refute the memory bias in recording the day at menarche. Subjects and methods: Girls from Santiago, Chile $(3,957)$ and Medellin, Colombia $(3,616)$ were asked about the date of birth and menarche. Results: In both cities, menarche occurred more commonly at birthday, Christmas-New Year, Day of the Independence, other holidays and days at vacation. The bias of memory was refuted because in both cities important holidays did not have excesses of menarche and some non-holidays had excesses; the distribution of menarches in the neighbor of peaks was often a left biased bell, with accessory peaks, and menarches/day over the expected mean (in agreement with the fiesta expectancy), and not a U shaped distribution (as expected by the memory bias); menarche distributed homogeneously on days of the week. Conclusions: The fiesta expectancy appears as a cause of the menarche rhythm. The bias of memory does not produce detectable effects. Evidence for recruitment of menarches and synergism between fiesta expectancy and vacations were found (Rev Méd Chile 2004; 132: 299-306).

(Key Words: Bias (Epidemiology); Data collection; Menarche; Sex maturation)

Recibido el 1 de septiembre, 2003. Aceptado en versión corregida el 21 de noviembre, 2003. Programa de Genética Humana, ICBM, Facultad de Medicina, Universidad de Chile.

A unque el ritmo anual de menarquia ha sido extensamente estudiado descriptivamente ${ }^{1-6}$, hay pocos estudios científicos deductivos que afirmen o refuten factores causales $3-5,7-9$. Con nuestro estudio descriptivo sobre menarquia ${ }^{6}$, iniciamos otros hipotético-deductivos que refutaron las variables climáticas, fotoperíodo y temperatura como

Correspondencia a: Carlos Y Valenzuela. Programa de Genética Humana, Instituto de Ciencias Biomédicas (ICBM), Facultad de Medicina, Universidad de Chile. Independencia 1027, Casilla 70061, Independencia, Santiago, Chile. Fax: (56-2) 7373158; Fonos: (56-2) 6786456-6786302. E-mail: cvalenzu@med.uchile.cl factores mayores de este ritmo anual $7,9-12$. Dimos evidencias sobre la distribución preferencial de las menarquias en meses de vacaciones y su depresión en meses de estudio ${ }^{12}$. Las influencias ambientales y el ritmo vacación-estudio se habían supuesto en trabajos anteriores ${ }^{4,5}$. También señalamos que había contradicción con estos efectos, ya que meses de exámenes escolares presentaban excesos de menarquia, como ocurría en diciembre en Chile o en abril en India ${ }^{11,12}$. Entonces, decidimos explorar la hipótesis que los días o períodos de días y no los meses fueran causas del ritmo anual de menarquia. Esta hipótesis plantea que la expectativa de fiesta 
(cumpleaños, descanso escolar, celebraciones nacionales o religiosas importantes para las niñas en esas edades) contribuye al desencadenamiento de la menarquia. Antitéticamente, esta hipótesis plantea que el trabajo escolar (estrés, preocupación) inhibe el desencadenamiento de la menarquia. Estudios seculares japoneses critican el mes de nacimiento y las vacaciones como asociados a excesos de menarquias ${ }^{3,13}$. Nuestra hipótesis explica esas discrepancias por cambios culturales en relación a las vacaciones y a la celebración del cumpleaños. Los meses contradictorios podrían incluir períodos de fiesta y no-fiesta. También someteremos a prueba el sesgo de memoria en sus dos componentes: i) el hipotético mejor recuerdo de la fecha de menarquia cuando ocurre en un día o período relevante; ii) el desplazamiento erróneo del día de menarquia desde los días cercanos a un día relevante hacia ese día relevante. $\mathrm{Si}$ es cierto que las niñas viven ritmos de expectativa de fiestano fiesta, que condicionan el ritmo de menarquia, se abre un campo fértil de investigación en las influencias psicosociales sobre los estados neuroendocrinos y en medicina psicosomática.

\section{MuESTRAS Y MÉTODOS}

Para abreviar, los meses serán denotados con las primeras 3 letras cuando sea necesario. Se aplicó, en los años 1990 y 1991, una encuesta a niñas escolares chilenas de Santiago; 3.957 menstruaban. En Medellín, Colombia, 3.616 niñas escolares, encuestadas en el año 1991, menstruaban. Una red de fechas importantes ayudaba a las niñas a recordar si su menarquia había o no sucedido en esas fechas o en relación a ellas. Para evitar el sesgo de memoria, se indicó a las niñas que la fecha (día, mes o año) de menarquia fuera consignada sólo si la recordaban certeramente; si no la recordaban, debían dejar el dato en blan$\mathrm{Co}^{7,9-12}$. En Chile, alumnos de postgrado entregaban las encuestas a las niñas con indicaciones para un auto-llenado. En Colombia, académicos entrenados vigilaron el llenado directamente. Esta diferencia en el método pudo causar que todas las niñas colombianas registraran su año de menarquia, en cambio el $1,8 \%$ de las chilenas no lo registró. Se descartaron los registros con errores clericales, incluidos aquellos cuyas edades de menarquia eran inferiores a 7,5 o superiores a 18,5 años (edades probables de ser erróneas). La proporción de niñas que recordaban el día, mes o año de menarquia fue menor mientras más tiempo había transcurrido entre este evento y la encuesta (según lo esperado); en este estudio no analizaremos esta relación. Tampoco estudiaremos la relación entre ritmo y edad de menarquia, ya que ésta está sesgada por los cursos escolares encuestados y porque incluye sólo a niñas que menstruaban al momento de la encuesta (niñas con pubertad más temprana), sesgo que no puede corregirse con este método. En Santiago (Chile) las vacaciones de verano ocurren desde mediados de diciembre a inicios de marzo. Las vacaciones de invierno son dos semanas en julio. Las primeras semanas de diciembre incluyen a menudo los exámenes finales. En Medellín (Colombia) hay vacaciones entre el 15 de junio y el 15 ó 20 de julio y a fin de año, entre el 20 de noviembre y el 31 de enero. Las fechas de fiestas en Colombia eran desconocidas para el autor al realizar este trabajo, lo que permitió probar la hipótesis que los excesos de menarquias denotan fiestas colombianas.

Reducción y prueba de hipótesis del sesgo de memoria. Este sesgo tiene 2 componentes. I) Las niñas recuerdan mejor el día de menarquia si ocurrió en un feriado, fecha notable (cumpleaños propio o familiar) o en domingo. Este sesgo es totalmente sobrepasado si usamos toda la muestra (las que recuerdan y no recuerdan la fecha). II) Las niñas pueden mover sus fechas de menarquias erróneamente desde los días cercanos a un día notable hacia ese día. Este movimiento generará una distribución en $\mathrm{U}$ circundando al día notable, con un gran exceso de menarquias en ese día. Ambos sesgos pueden ser refutados si hay feriados que no presentan excesos de menarquia y si hay no-feriados que los presentan 0 , si hay una curva en campana en vez de en U.

Coincidencia con el día de cumpleaños. Se obtuvo la variable D (DIA), que es la distancia en días calendarios (variable $d=$ día), en un año de 365 días, entre el día de menarquia y el día de nacimiento. La variable va desde $-182 \mathrm{D}$ a $+182 \mathrm{D}$; los números negativos indican que la menarquia 
ocurrió antes que el nacimiento y los positivos la situación inversa; 0D indica la coincidencia menarquia-cumpleaños. El número de menarquias se indica por la variable $M$, entonces $(-65 D=10 M)$ indicará que 65 días antes del cumpleaños ocurrieron 10 menarquias.

Estadística. La distribución azarosa esperada del número de menarquias $(\mathrm{M})$ en un día calendario (d, M/d) o en relación al cumpleaños (D, M/D) es una binomial. Si se toma toda la muestra $\left(\mathrm{N}_{\mathrm{T}} 0\right.$ total no-sesgado), el promedio esperado de $\mathrm{M} / \mathrm{d} 0$ $\mathrm{M} / \mathrm{D}$ es $\mathrm{N}_{\mathrm{T}} / 365$ con varianza $\mathrm{N}_{\mathrm{T}}\left(364 / 365^{2}\right)$. Si se usa el total sesgado $\mathrm{N}_{\mathrm{D}}$ (las que recuerdan día o mes de menarquia) éste reemplaza a $\mathrm{N}_{\mathrm{T}}$. Para 365 días los niveles de confianza se eligen con $\alpha / 2=$ $1 /(2 \times 365)=0,00137$ de modo de encontrar a lo más un $\mathrm{d}$ o $\mathrm{D}$ con $\mathrm{M}$ igual o más extremo que el límite inferior (Lnf) o superior (LSup) del intervalo de confianza (IC) definido por ese nivel. Este $\alpha /$ 2 corresponde a $\mathrm{z}_{\mathrm{IC}}=2.993 \mathrm{DT}$ (Desviaciones Típicas). Para meses el IC se construye similarmente (31 en vez de 365), así $\alpha / 2=1 /(2 \times 31)=$ $0,01613, \mathrm{z}_{\mathrm{IC}}=2,143 \mathrm{DT}$. Otros análisis se indican en resultados.

\section{RESULTADOS Y DISCUSIÓN}

\section{Análisis de la coincidencia con el cumpleaños}

La muestra chilena. Sin los errores clericales quedaron 3.777 niñas $\left(\mathrm{N}_{\mathrm{T}}\right)$, de las que $98,2 \%$ recordaron el año, 88,9\% recordaron año y mes y, $65,6 \%$ recordaron día, mes y año de menarquia. El promedio $\left(\mathrm{P}_{\mathrm{M}}\right)$ y DT insesgados fueron $\mathrm{P}_{\mathrm{M}}=10,36$ M/D y DT=3,214; el IC para $365 \mathrm{D}$ (ver métodos) dio $\operatorname{Lnf}=0,74(1 \mathrm{M} / \mathrm{D})$, LSup=19,98 (19 M/D). No hubo $\mathrm{D}$ con $0 \mathrm{M}$, cerca de $19 \mathrm{M}$ o más se encontró $-3 \mathrm{D}=18 \mathrm{M} ;-2 \mathrm{D}=30 \mathrm{M} ;-1 \mathrm{D}=22 \mathrm{M} ; 0 \mathrm{D}=49 \mathrm{M} ;+1 \mathrm{D}=$ $26 \mathrm{M} ;+2 \mathrm{D}=22 \mathrm{M} ;+3 \mathrm{D}=18 \mathrm{M}$; que es el período alrededor del cumpleaños, flanqueado por $-4 \mathrm{D}=$ $10 \mathrm{M} \mathrm{y}+4 \mathrm{D}=8 \mathrm{M}$ (entre Linf y LSup). El resto de D se distribuyó según la binomial esperada. Con parámetros sesgados (las que recuerdan el día de menarquia) el promedio fue $6,79 \mathrm{M} / \mathrm{D}, \mathrm{DT}=2,601$, $\operatorname{Lnf}=0,0(0 \mathrm{M} / \mathrm{D}), \operatorname{LSup}=14,55(14 \mathrm{M} / \mathrm{D}) . \mathrm{Al}$ igual que la muestra insesgada, el intervalo $-3 \mathrm{D}$ a +3D mostró más de $15 \mathrm{M} / \mathrm{D}$, revelando que la acumulación de menarquias cerca del cumpleaños excede cualquier sesgo I de memoria. Si fuera cierto el sesgo II, la distribución en torno a OD debería ser en $\mathrm{U}$; se dio lo contrario; una distribución en campana con un segundo pico en $-2 \mathrm{D}$ sesgada a la izquierda (70M antes y $66 \mathrm{M}$ después de 0D), lo que indica más bien reclutamiento de menarquias alrededor del cumpleaños (de acuerdo a la expectativa de fiesta).

La muestra colombiana. Las 3.616 niñas recordaban el año, 99,6\% recordaban el año y mes y $62,1 \%$ recordaban el año, mes y día de menarquia. El promedio insesgado fue $9,91 \mathrm{M} / \mathrm{D}$ con $\mathrm{DT}=$ 3,143; el IC tuvo $\operatorname{Lnf}=0,5$ (1 M/D) y $\operatorname{LSup}=19,3$ (19 M/D). No se encontró ningún D con 0M y sólo $0 \mathrm{D}=36 \mathrm{M}$ fue significativo con este criterio exigente. El intervalo alrededor de $0 \mathrm{D}$ fue $-3 \mathrm{D}=17 \mathrm{M}$, $2 \mathrm{D}=14 \mathrm{M},-1 \mathrm{D}=11,0 \mathrm{D}=36 \mathrm{M}, 1 \mathrm{D}=14 \mathrm{M}, 2 \mathrm{D}=11 \mathrm{M}$, $3 \mathrm{D}=7 \mathrm{M}$; flanqueado por $-4 \mathrm{D}=5 \mathrm{M}$ y $+4 \mathrm{D}=8 \mathrm{M}$. El resto se ajustó a la binomial. El $\mathrm{P}_{\mathrm{M}} \mathrm{y}$ DT sesgados fueron $\mathrm{P}_{\mathrm{M}}=6,15 \mathrm{M} / \mathrm{D}$ y $\mathrm{DT}=2,477$, con $\operatorname{Lnf}=-1,3$ (0) y LSup $=11,62(11) \mathrm{M} / \mathrm{D}$. Fueron significativos con estos parámetros -3D, -2D, 0D, 1D y 3D en torno al cumpleaños y, en el límite de significación $-66 \mathrm{D}=12 \mathrm{M}, 5 \mathrm{D}=12 \mathrm{M}, 153 \mathrm{D}=12 \mathrm{M}$ y $158 \mathrm{D}=$ $12 \mathrm{M}$, probablemente por azar. Como en Santiago, el cumpleaños se halla en una distribución en campana (expectativa de fiesta) y no en U (sesgo de memoria). Esta campana también es sesgada a izquierda (42 versus $32 \mathrm{M}$ ) con pico secundario en $-3 \mathrm{D}$, lo que indica reclutamiento.

Análisis de los días calendarios. Como los meses del año posteriores a los meses en que se encuestó estaban subrepresentados, se excluyeron en Chile las menarquias de los años 1990 y 1991 según correspondía y, en Colombia, las menarquias ocurridas en 1991.

La muestra chilena. La Tabla 1 muestra la distribución mensual de las menarquias de esta muestra (años 1990-1) y de una muestra anterior (1978)6,7. Para esta muestra se incluyen los estimadores de parámetros de la muestra mensual no sesgada (MMN, niñas que recordaban o no el día de menarquia), el promedio mensual esperado, DT, Lnf y LSup del IC mensual (ver métodos). La muestra se redujo a 3.211 niñas (NT), que dieron 
un promedio de $8,77 \mathrm{M} / \mathrm{d}(\mathrm{DT}=2,958)$, $\operatorname{Lnf}=-0,08$ $(0 \mathrm{M} / \mathrm{d}), \operatorname{LSup}=17,63(17 \mathrm{M} / \mathrm{d})$. De estas niñas 3.141 recordaron el año, 2.811 el mes y el año y 2.016 el día, mes y año (Tabla 1).

En ambas muestras (1978 y 1990-1) hubo excesos de menarquias en diciembre, enero y febrero y deficiencias en los otros meses notables en mayo y abril (análisis mensual ya presentado ${ }^{7}$ ). Ambas muestras también difieren. El orden de excesos fue en 1978 Dic $>$ Ene $>$ Feb y Jun $>$ Ago $>$ Jul, en cambio fue Feb $>$ Dic $>$ Ene y Jul $>$ Ago $>$ Jun en 1990-91. Febrero 1978 presentó una menor proporción de menarquias que febrero 1990-1. La heterogeneidad de $\mathrm{M} / \mathrm{d}$ dentro de un mes (períodos de fiesta y no-fiesta) se estimó por una prueba $\chi^{2}$ con (n-1) grados de libertad $(\mathrm{n}=$ días del mes 0 año), ya que la variable $\left[(n-1) s^{2} / \sigma^{2}\right]$ así se distribuye. Excepto julio y agosto, todos los meses fueron significativamente heterogéneos $\left(10^{-9}<\mathrm{P}<0,027\right)$. La heterogeneidad en todo el año fue muy significativa $\left(\mathrm{P}<10^{-15}\right)$. Los valores de la Tabla 1 permiten detectar días con números relevantes de menarquia que se muestran en la Tabla 2.
Los estimadores mensuales sesgados dieron excesos significativos en días o períodos: Navidad-(Año Nuevo) (diciembre 20, 22, 24, 25, 31 y enero 1, 5); Fiestas Patrias (septiembre 15, 16, 17, 18); el día con mayor exceso fue febrero 14 (día de los enamorados) que no es feriado y ocurre en vacaciones. Mayo 21 (día patrio) tiene un exceso significativo y contrasta con mayo 1 que siendo feriado no tiene exceso de menarquias (refuta sesgo de memoria). Los otros excesos significativos son pequeños, excepto febrero 28. En torno a Navidad hubo una distribución en campana con pico en diciembre 24 y no 25 (refuta sesgo de memoria), sesgada a izquierda y 3 picos accesorios (diciembre 20, 22 y 25), lo que hace imposible la explicación por sesgo de memoria y afirma fuertemente la expectativa de fiesta con reclutamiento de menarquias (aproximación dentada desde la izquierda). El sesgo de memoria se refuta también porque importantes feriados no tuvieron excesos de menarquias: mayo 1, septiembre 19 (festividad patria donde se realiza la Parada Militar), octubre 12, noviembre 1, diciembre 8;

Tabla 1. D istribución mensual de menarquias. Niñas chilenas. Muestras de 1978 y 1990-1991, con indicadores para ubicar días notables

\begin{tabular}{|c|c|c|c|c|c|c|c|c|c|c|}
\hline \multirow[b]{3}{*}{ Mes } & \multirow{2}{*}{\multicolumn{2}{|c|}{$\begin{array}{c}\text { Muestra de } \\
1978\end{array}$}} & \multirow{2}{*}{\multicolumn{2}{|c|}{ MMS }} & \multicolumn{3}{|c|}{ Muestra de 1990-1991 } & \multirow{2}{*}{\multicolumn{2}{|c|}{ IC }} & \multirow{3}{*}{$\mathrm{MMN}$} \\
\hline & & & & & $\mathrm{P}_{\mathrm{M}}$ & \multicolumn{2}{|c|}{ DT } & & & \\
\hline & Esp & Obs & Esp & Obs & $\mathrm{M} / \mathrm{d}$ & Esp & Obs & $\mathrm{L} \operatorname{Inf}$ & L Sup & \\
\hline Ene & 200,4 & 304 & 171,1 & 263 & 8,48 & 2,87 & 6,06 & $2,3(3)$ & $14,6(14)$ & 363 \\
\hline Feb & 182,6 & 294 & 155,9 & 296 & 10,48 & 3,18 & 7,22 & $3,7(4)$ & 17,3 (17) & 439 \\
\hline Mar & 200,4 & 165 & 171,1 & 136 & 4,39 & 2,06 & 3,86 & $-0,0(0)$ & $8,8(8)$ & 199 \\
\hline $\mathrm{Abr}$ & 193,9 & 112 & 165,6 & 82 & 2,73 & 1,63 & 2,00 & $-0,8(0)$ & $6,2(6)$ & 118 \\
\hline May & 200,4 & 101 & 171,1 & 87 & 2,81 & 1,65 & 2,31 & $-0,7(0)$ & $6,3(6)$ & 110 \\
\hline Jun & 193,9 & 186 & 165,6 & 107 & 3,57 & 1,86 & 2,46 & $-0,5(0)$ & $7,4(7)$ & 145 \\
\hline Jul & 200,4 & 152 & 171,1 & 136 & 4,38 & 2,06 & 1,88 & $-0,0(0)$ & $8,8(8)$ & 209 \\
\hline Ago & 200,4 & 164 & 171,1 & 120 & 3,87 & 1,94 & 1,83 & $-0,3(0)$ & $8,0(8)$ & 166 \\
\hline Sep & 193,9 & 184 & 165,6 & 172 & 5,73 & 2,35 & 4,54 & $0,7(1)$ & $10,8(10)$ & 237 \\
\hline Oct & 200,4 & 191 & 171,1 & 157 & 5,06 & 2,21 & 2,95 & $0,3(1)$ & $9,8(9)$ & 200 \\
\hline Nov & 193,9 & 196 & 165,6 & 145 & 4,83 & 2,16 & 2,15 & $0,2(1)$ & $9,5(9)$ & 220 \\
\hline Dic & 200,4 & 312 & 171,1 & 315 & 10,16 & 3,14 & 6,97 & $3,4(4)$ & $16,9(16)$ & 405 \\
\hline Tot & & 2.361 & & 2.016 & 5,52 & 2,35 & 4,87 & $1,5(0)$ & $12,5(12)$ & 2.811 \\
\hline
\end{tabular}

$\mathrm{M}=$ menarquias; $\mathrm{P}_{\mathrm{M}}=$ promedio $\mathrm{M}$ / día; $\mathrm{DT}=$ Desviación Típica; $\mathrm{IC}=$ intervalo de confianza; $\mathrm{MMS}=$ muestra mensual sesgada (niñas que recordaban el día y mes de menarquia); $M M N=$ muestra mensual no-sesgada (niñas que recordaban el mes con y sin recordar el día); Esp=esperado; Obs=observado; L=límite; Inf=inferior; Sup=superior. 
Tabla 2. D ías notables en la muestra chilena de 1990-1

\begin{tabular}{|c|c|c|c|c|c|}
\hline \multicolumn{6}{|c|}{ Días notables con estimadores mensuales sesgados (IC en Tabla 1) } \\
\hline Enero & Febrero & Marzo & Abril & Mayo & Junio \\
\hline $1=33 \mathrm{M}+$ & $14=43 \mathrm{M}+$ & $3=14 \mathrm{M}+$ & $12=7 \mathrm{M}+$ & $10=9 \mathrm{M}+$ & $12=11 \mathrm{M}+$ \\
\hline $5=15 \mathrm{M}+$ & $28=18 \mathrm{M}+$ & $5=15 \mathrm{M}+$ & $15=8 \mathrm{M}+$ & $21=11 \mathrm{M}+$ & $13=9 \mathrm{M}+$ \\
\hline $\begin{array}{l}15=15 \mathrm{M}+ \\
21=1 \mathrm{M}-\end{array}$ & $\begin{array}{l}3=3 \mathrm{M}- \\
9=3 \mathrm{M}-\end{array}$ & $\begin{array}{l}7=13 \mathrm{M}+ \\
\text { Septiembre }\end{array}$ & Octubre & \multicolumn{2}{|c|}{ Diciembre } \\
\hline $22=2 \mathrm{M}-$ & & $15=12 \mathrm{M}+$ & $5=16 \mathrm{M}+$ & $20=17 \mathrm{M}+$ & $22=22 \mathrm{M}+$ \\
\hline $23=2 \mathrm{M}-$ & & $16=13 \mathrm{M}+$ & & $24=30 \mathrm{M}+$ & $25=23 \mathrm{M}+$ \\
\hline $\begin{array}{l}29=2 \mathrm{M}- \\
\text { Julio }\end{array}$ & Agosto & $\begin{array}{l}17=13 \mathrm{M}+ \\
18=23 \mathrm{M}+\end{array}$ & $\begin{array}{c}\text { Noviembre } \\
----\end{array}$ & $\begin{aligned} 31 & =26 \mathrm{M}+ \\
9 & =3 \mathrm{M}-\end{aligned}$ & $\begin{array}{c}4=3 \mathrm{M}- \\
10=3 \mathrm{M}\end{array}$ \\
\hline---- & ---- & $6=0 \mathrm{M}-$ & & & \\
\hline \multicolumn{6}{|c|}{ Días notables con estimadores anuales insesgados (IC $=0-17 \mathrm{M} / \mathrm{d}$ ) } \\
\hline Enero & & Febrero & Septiembre & \multicolumn{2}{|c|}{ Diciembre } \\
\hline $1=33 \mathrm{M}+$ & & $\begin{array}{l}14=43 \mathrm{M}+ \\
28=18 \mathrm{M}+\end{array}$ & $18=23 \mathrm{M}+$ & $\begin{array}{l}22=22 \mathrm{M}+ \\
25=23 \mathrm{M}+\end{array}$ & $\begin{array}{l}24=30 \mathrm{M}+ \\
31=26 \mathrm{M}+\end{array}$ \\
\hline \multicolumn{6}{|c|}{ Días Notables. Estimadores mensuales insesgados (MMN) Solo + } \\
\hline Enero & Febrero & Marzo & Abril & Mayo & Junio \\
\hline $1=33 \mathrm{M}$ & $14=43 \mathrm{M}$ & $3=14 \mathrm{M}$ & ---- & $10=9 \mathrm{M}$ & $12=11 \mathrm{M}$ \\
\hline Julio & Agosto & $5=15 \mathrm{M}$ & Septiembre & $21=11 \mathrm{M}$ & $15=10 \mathrm{M}$ \\
\hline---- & ---- & $7=13 \mathrm{M}$ & $18=23 \mathrm{M}$ & \multicolumn{2}{|c|}{ Diciembre } \\
\hline Octubre & Noviembre & & & $22=22 \mathrm{M}$ & $24=30 \mathrm{M}$ \\
\hline $5=16 \mathrm{M}$ & ---- & & & $25=23 \mathrm{M}$ & $31=26 \mathrm{M}$ \\
\hline
\end{tabular}

+ y - = más y menos M/d que lo esperado.

Tabla 3. D istribución mensual de menarquias. Niñas colombianas. M uestra de 1991, con indicadores para ubicar días notables

\begin{tabular}{|c|c|c|c|c|c|c|c|c|}
\hline \multirow[b]{2}{*}{ Mes } & \multicolumn{2}{|c|}{ MMS } & \multirow{2}{*}{$\begin{array}{c}\mathrm{P}_{\mathrm{M}} \\
\mathrm{M} / \mathrm{d}\end{array}$} & \multicolumn{2}{|c|}{ DT } & \multicolumn{2}{|c|}{ IC } & \multirow[t]{2}{*}{$\mathrm{MMN}$} \\
\hline & Esp & Obs & & Esp & Obs & $\mathrm{L} \operatorname{Inf}$ & L Sup & \\
\hline Ene & 158,6 & 173 & 5,58 & 2,324 & 3,180 & $0,60(1)$ & $10,56(10)$ & 249 \\
\hline Feb & 144,6 & 113 & 3,90 & 1,964 & 2,468 & $-0,21(0)$ & 8,21 (8) & 159 \\
\hline Mar & 158,6 & 91 & 2,94 & 1,685 & 1,900 & $-0,68(0)$ & $6,55(6)$ & 166 \\
\hline $\mathrm{Abr}$ & 153,5 & 98 & 3,27 & 1,777 & 1,861 & $-0,54(0)$ & 7,08 (7) & 190 \\
\hline May & 158,6 & 131 & 4,23 & 2,022 & 2,349 & $-0,11(0)$ & $8,56(8)$ & 184 \\
\hline Jun & 153,5 & 116 & 3,87 & 1,933 & 2,349 & $-0,28(0)$ & 8,01 (8) & 225 \\
\hline Jul & 158,6 & 206 & 6,65 & 2,536 & 4,186 & $1,21(2)$ & $12,08(12)$ & 510 \\
\hline Ago & 158,6 & 109 & 3,52 & 1,845 & 2,138 & $-0,44(0)$ & $7,47(7)$ & 184 \\
\hline Sep & 153,5 & 131 & 4,37 & 2,055 & 2,041 & $-0,04(0)$ & 8,77 (8) & 184 \\
\hline Oct & 158,6 & 170 & 5,48 & 2,304 & 2,435 & $0,55(1)$ & $10,42(10)$ & 239 \\
\hline Nov & 153,5 & 198 & 6,60 & 2,526 & 2,917 & $1,19(2)$ & $12,01(12)$ & 335 \\
\hline Dic & 158,6 & 333 & 10,74 & 3,224 & 5,869 & $3,83(4)$ & 17,65 (17) & 507 \\
\hline Tot & & 1.869 & 5,11 & 2,257 & 3,676 & $-1,65(0)$ & $11,86(11)$ & 3.132 \\
\hline
\end{tabular}

Nomenclatura como en Tabla 1. 
además que varios no-feriados lo tuvieron como fueron febrero 14 y 28 los previos a Navidad y al 18 de septiembre.

Los meses con grandes varianzas podrían contener períodos de fiesta y no-fiesta. Las dos primeras semanas de diciembre son estudio $\mathrm{y}$ exámenes finales. El promedio en el periodo diciembre $1-18$ fue $6,0 \mathrm{M} / \mathrm{d}(\mathrm{DT}=2,48)$ y en el periodo diciembre $19-31$ fue $15,9 \mathrm{M} / \mathrm{d}(\mathrm{DT}=7,19)$; diferencia muy significativa $\left(t_{29}=17,7 ; P<10^{-12}\right)$. La primera semana de marzo es de vacaciones con promedio $9,1 \mathrm{M} / \mathrm{d}(\mathrm{DT}=4,63)$, las restantes son de estudio y el promedio fue $3,0 \mathrm{M} / \mathrm{d}$ (DT=2,36); diferencia significativa $(\mathrm{P}=0,002)$. La hipótesis del aumento de varianza por mezcla de períodos de fiesta y no-fiesta se confirma.

La muestra de Colombia. Sin las menarquias de 1991 (año de encuesta) quedaron 3.146 niñas que recordaban el año, 3.132 (99,6\%) el año y mes y $1.869(59,4 \%)$ el día, mes y año de menarquia. El promedio anual insesgado fue $8,60 \mathrm{M} / \mathrm{d}$ (DT= 2,928); El IC tuvo $\operatorname{Lnf}=0,17$ (0 M/d) y $L S u p=17,36$ $(17 \mathrm{M} / \mathrm{d})$. La Tabla 3 muestra la distribución y parámetros mensuales relevantes (Tabla 3 ).
Excesos significativos en diciembre, julio y noviembre y depresiones en marzo, abril, agosto, junio, febrero y mayo coincidieron con los descritos para esta muestra sin descartar errores clericales y menarquias en $1991^{10}$. Todos los meses, excepto septiembre, tuvieron varianzas mayores que las esperadas y significativas en diciembre, julio, enero, febrero y mayo $\left(10^{-12}<\mathrm{P}\right.$ $<0,047)$. Con los parámetros de la Tabla 3 se determinaron los días con número de menarquia más conspicuos (Tabla 4). El calendario de días de fiesta lo obtuvimos con nuestros colegas colombianos.

Los días más relevantes se relacionaron con el período Navidad-Año Nuevo, diciembre 8 y julio 20. Diciembre 8 es la fiesta católica más relevante para las niñas, porque continúan haciendo su Primera Comunión en ese día. Es notable que en Chile la Iglesia Católica discontinuó esa práctica desde hace más de 20 años. Además diciembre 8 está en período de estudios en Chile y de vacaciones en Colombia, lo que indica sinergismo entre la expectativa de fiesta y las vacaciones. Julio 20 es el Día Patrio en Colombia. Enero 9 está cerca de Epifanía que es celebrada con el domingo más

Tabla 4. D ías notables en la muestra colombiana de 1991

\begin{tabular}{|c|c|c|c|c|c|}
\hline \multicolumn{6}{|c|}{ Días notables con estimadores mensuales sesgados (IC en Tabla 3) } \\
\hline Enero & Febremo & Marzo & Abril & Mayo & Junio \\
\hline $\begin{array}{l}1=13 \mathrm{M}+ \\
9=12 \mathrm{M}+\end{array}$ & $1=9 \mathrm{M}+$ & ---- & $12=8 \mathrm{M}+$ & $13=11 \mathrm{M}+$ & $\begin{array}{c}28=10 \mathrm{M}+ \\
\text { Diciembre }\end{array}$ \\
\hline Julio & Agosto & Septiembre & Octubre & Noviembre & $8=28 \mathrm{M}+$ \\
\hline $16=13 \mathrm{M}+$ & $12=9 \mathrm{M}+$ & $12=9 \mathrm{M}+$ & $27=11 \mathrm{M}+$ & $22=14 \mathrm{M}+$ & $16=23 \mathrm{M}+$ \\
\hline $\begin{array}{l}20=22 \mathrm{M}+ \\
22=1 \mathrm{M}- \\
27=1 \mathrm{M}- \\
31=1 \mathrm{M}-\end{array}$ & $25=10 \mathrm{M}+$ & ---- & $31=12 \mathrm{M}+$ & $25=13 \mathrm{M}+$ & $\begin{array}{l}22=18 \mathrm{M}+ \\
24=19 \mathrm{M}+ \\
28=22 \mathrm{M}+ \\
31=19 \mathrm{M}+\end{array}$ \\
\hline \multicolumn{6}{|c|}{ Días notables con estimadores anuales insesgados $(\mathrm{CI}=0-17 \mathrm{M} / \mathrm{d})$} \\
\hline Julio & & & & Diciembre & \\
\hline $20=22 \mathrm{M}$ & & & $\begin{array}{c}8=28 \mathrm{M}+ \\
24=19 \mathrm{M}+\end{array}$ & $\begin{array}{l}16=23 \mathrm{M}+ \\
28=22 \mathrm{M}+\end{array}$ & $\begin{array}{l}22=18 \mathrm{M}+ \\
31=19 \mathrm{M}+\end{array}$ \\
\hline $\begin{array}{c}\text { Días notables. } \\
\text { Diciembre } \\
8=28 \mathrm{M}\end{array}$ & timadores me & insesgados ( & lo+ & & \\
\hline
\end{tabular}

Nomenclatura como en Tabla 2. 
cercano. Mayo 13 es la fiesta de la Vingen de Fátima y cerca del segundo domingo de mayo (día de la Madre). Junio 28 es la víspera de San Pedro y San Pablo. Entre agosto 7-12 hay una fiesta semanal de las flores (antigua fiesta campesina) y también se celebra la Batalla de Boyacá (Fiesta Patria en Colombia). Octubre 31 es la víspera de Todos los Santos y se ha introducido la celebración anglosajona de Halloween. Diciembre 28 no es feriado, es día de los inocentes. Vemos que varios días de fiesta o feriados tienen excesos de menarquias, pero otros no lo tienen (mayo 1, octubre 12, agosto 15), además varios días con excesos no corresponden a feriado o fiesta. Se refuta en Colombia la hipótesis del sesgo de memoria al igual que en Chile. Más aún, las menarquias en torno a Navidad también se presentaron en campana sesgada a izquierda y picos accesorios (diciembre 22, diciembre 28). Aquí, diciembre 25 ni siquiera mostró un exceso significativo $(9 \mathrm{M}$, imposible según sesgo de memoria). Julio del $1 \mathrm{al}$ 20, tiempo de vacaciones tuvo $\mathrm{P}_{\mathrm{M}}=8,4 \mathrm{M} / \mathrm{d}, \mathrm{DT}=$ 4,17, en el resto, de estudio, $\mathrm{P}_{\mathrm{M}}=3,5 \mathrm{M} / \mathrm{d}$ y $\mathrm{DT}=$ 2,21; diferencia significativa $(\mathrm{P}=0,00034)$ que afirma la hipótesis de heterogeneidad fiesta-no fiesta dentro del mes.

Análisis de los días de la semana. En la muestra chilena la distribución fue: domingo 366M; lunes 382M; martes 354M; miércoles 340M; jueves 350M; viernes 333M; sábado 352M; total 2.477. La distri-

\section{REFERENCIAS}

1. VALSIK JA. The seasonal rhythm of menarche. A review. Hum Biol 1965; 37: 75-90.

2. BojLen $K$, Weis Bentzon W. Seasonal variation in the occurrence of menarche. Dan Med Bull 1974; 21: 161-8.

3. Nakamura I, Shimura M, Nonaka K, Miura T. Changes of recollected menarcheal age and month among women in Tokyo over a period of 90 years. Ann Hum Biol 1986; 13: 547-54.

4. Wolanski N, Dickinson F, Siniarska A. Seasonal rhythm of menarche as a sensitive index of living conditions. Stud Hum Ecol 1993; 11: 171-91. bución fue homogénea $\left(\chi_{6}^{2}=4,45, \mathrm{P}=0,62\right)$. En Colombia la distribución fue: domingo 322M; lunes 315M; martes 306M; miércoles 322M; jueves $316 \mathrm{M}$; viernes 331M; sábado 334M; total 2.246. La distribución fue homogénea $\left(\chi_{6}^{2}=1,74, \mathrm{P}=0,94\right)$. En ambas muestras no se dio el exceso de menarquias esperado para el día domingo.

Se concluye que la expectativa de fiesta se correlaciona fuertemente con los excesos de menarquia, que se dan generalmente no en un día aislado sino que en un período en torno a un día pico. Hay indicios sistemáticos de un proceso de reclutamiento de menarquias (sesgo a izquierda y picos accesorios al principal). Puede ser que el período (expectativa) de no-fiesta al incluir más estrés inhibiría la menarquia que necesita más de 10 días para desencadenarse (como cualquier menstruación). La expectativa de fiesta relaja a la niña y, entonces, aquellas que están más «maduras» presentan su primera menstruación. El sesgo de memoria se refuta por todos los criterios: acumulación heterogénea en feriados; excesos en noferiados; distribución de menarquias en campana en torno a un pico (según expectativa de fiesta) y no en U (según sesgo de memoria); el domingo no presentó exceso de menarquias. Además, el método disminuye el sesgo al requerir la fecha sólo si había seguridad y, al usar toda la muestra (nosesgada en memoria) los resultados fundamentales no variaron. El sesgo de memoria resulta ser más bien un mito de efectos indetectables.

5. GueRESI P. Monthly distribution of menarche in three provinces of North Italy. Ann Hum Biol 1997; 24: 157-68.

6. Patri A, Valenzuela CY, Morales I, Saavedra I, FigueroA L. Age at menarche of girls from public schools of the Northern Area of Santiago. Cuad Méd Soc (Santiago, Chile) 1980; 21: $12-20$.

7. ValenZuela CY, NúÑEZ E, TAPiA C. Month at menarche: a re-evaluation of the seasonal hypothesis. Ann Hum Biol 1991; 18: 383-93.

8. CoHen P. Month at menarche: a re-evaluation of the seasonal hypothesis. Ann Hum Biol 1993; 20: 198-200. 
9. Valenzueia CY, NúÑEZ E, TAPiA C. Month at menarche: a re-evaluation of the seasonal hypothesis. Annals Human Biology 1993; 20: 200-2.

10. Valenzuela CY, Pineda N, Olarte G, Vásquez G. Annual rhythm of menarche in Medellin, Colombia. Rev Méd Chil 1996; 124: 437-41.

11. Valenzuela CY, SRikumari CR, Gopinath PM, Ghose N, Gajalakshmi P, Csoknyay J. New evidence of non seasonal factors in the menarche rhythm. Biol Res 1996; 29: 245-51.
12. Vaienzueia CY, SRikumari CR, Csoknyay J, Pineda N. Annual menarche and scholar rhythms. Rev Méd Chil 1999; 127: 143-50.

13. Miura T, Nakamura I, Nonaka K, Shimura $M$. Correlation between month of menarche and month of birth. Gynecology 1987; 240: 195-200.

Agradecimientos

A las niñas que participaron en los estudios y a mis colegas de India, Hungría, Colombia y Chile con quienes realicé los estudios previos. 